\title{
Robust self-triggered MPC with adaptive prediction horizon for perturbed nonlinear systems
}

\author{
Zhongqi Sun, Li Dai, Kun Liu, Dimos V. Dimarogonas, and Yuanqing Xia, Senior Member, IEEE
}

\begin{abstract}
This paper proposes a robust self-triggered model predictive control (MPC) with an adaptive prediction horizon scheme for constrained nonlinear discrete-time systems subject to additive disturbances. At each triggering instant, the controller provides an optimal control sequence by solving an optimal control problem (OCP), and at the same time, determines the next triggering time and prediction horizon. By implementing the algorithm, the average sampling frequency is reduced and the prediction horizon is adaptively decreased as the system state approaches a terminal region. Meanwhile, an upper bound of performance loss is guaranteed when compared with a nominal periodic sampling MPC. Feasibility of the OCP and stability of the closed-loop system are established. Simulation results verify the effectiveness of the scheme.
\end{abstract}

Index Terms-Self-triggered control, model predictive control (MPC), adaptive prediction horizon, nonlinear systems.

\section{INTRODUCTION}

M ODEL predictive control (MPC) has the advantages of explicitly handling input and state constraints and optimizing the performance [1]. Generally, traditional MPC requires a quite heavy computation, especially for nonlinear systems, to solve an optimization control problem (OCP) at each step. This may prevent its application to "fast" systems such as unmanned ground vehicles, quadrotors and servo systems, etc. Therefore the design of MPC with reduced computational load is an urgent demand for its application.

Event-triggered and self-triggered MPC are studied in [2][6] which aim at achieving a better trade-off between system performance and resource saving. For instance, an eventtriggered MPC for continuous-time nonlinear systems with constraints is developed in [2] based on Lyapunov theory. By checking the deviation between the actual state and the

The first, second, third and the fifth author were supported by the National Natural Science Foundation Projects of International Cooperation and Exchanges under Grant 61720106010, the Beijing Natural Science Foundation under Grant 4161001, Grant Z170039 and Grant 4182057, the National Natural Science Foundation under Grant 61603041, Grant 61803033, Grant 61836001 and Grant 61873034, the National Key Research and Development Program of China under Grant 2018YFB1003700, the Foundation for Innovative Research Groups of the National Natural Science Foundation of China under Grant 61621063. The fourth author was supported by the Swedish Research Council (VR), Knut och Alice Wallenberg foundation (KAW), and the H2020 ERC Starting Grant BUCOPHSYS.(Corresponding author: Yuanqing Xia.)

Zhongqi Sun, Li Dai, Kun Liu and Yuanqing Xia are with the Key Laboratory of Complex System Control and Decision, School of Automation, Beijing Institute of Technology, Beijing 100081, China (e-mails: sunzhongqi12@gmail.com; daili1887@gmail.com; kunliubit@bit.edu.cn; xia_yuanqing@bit.edu.cn). Dimos V. Dimarogonas is with the School of Engineering, KTH Royal Institute of Technology, Stockholm SE-100 44, Sweden and also with the KTH Center of Autonomous Systems, Stockholm SE-100 44, Sweden (e-mail: dimos@kth.se). predicted one, an event-triggered mechanism is developed in [3] to reduce the update rate. The authors of [4] introduce a self-triggered strategy for linear systems based on performance levels described by a quadratic discounted cost. A robust selftriggered tube-based MPC is proposed in [5], in which a parameter is introduced into the cost function allowing a tradeoff between the performance and the sampling rate. A selftriggered MPC for nonlinear input-affine networked systems is studied in [6] by selecting sampling intervals adaptively. The optimal control trajectory is discretized into several control samples such that the control signal can be transmitted over the network. The difference between event-triggered and selftriggered MPC lies in that event-triggered MPC requires constant measurement of the current states, while in self-triggered MPC, the next update time is computed at the previous one. Therefore, the self-triggered mechanism has the advantage of requiring less states information, whereas it is susceptible to uncertainties and requires more computations when compared to the event-triggered control scheme [5].

The approaches discussed above are able to alleviate the computation burden, but it only through the reduction in the frequency of solving the OCP. The computational complexity at each update remains high, because the prediction horizon is usually a fixed constant. In this paper, we develop a robust selftriggered MPC with an adaptive prediction horizon scheme to relieve the heavy computation burden for disturbed discretetime nonlinear systems. On the one hand, the update time is computed in a self-triggered fashion to reduce the frequency of solving the OCP. On the other hand, the prediction horizon decreases adaptively as the system state approaches a terminal region. This leads to the reduction of the OCP dimension thereby reducing the computational complexity at each sampling time. A similar idea on shrinking the prediction horizon is employed in [7] for continuous-time systems, in which the event-triggered condition is detected only at certain time instants. Different from [7], we focus on discrete-time nonlinear systems, use a self-triggered strategy and guarantee a suboptimal performance. In addition, the prediction horizon is decreased adaptively. These features may render the strategy more suitable for practical applications. The main contributions of this paper are summarized as follows: 1) a novel robust constraint tightening scheme for nonlinear discrete-time systems is developed that guarantees robustness of MPC; 2) a self-triggered mechanism and a prediction horizon update strategy are presented which save the computing resources in two ways: decreasing the frequency of solving the OCP, and reducing the complexity of each OCP; and 3) recursive feasibility and input-to-state stability (ISS) are established 
based on a dual-mode MPC strategy.

The remainder of this paper is organized as follows. Section II contains notations and preliminary results. We formulate the control problem and control objective in Section III. A robust self-triggered MPC with adaptive prediction horizon scheme is developed in Section IV. In Section V, the efficiency of the scheme is verified by a simulation example. Section VI concludes the paper.

\section{NOTATION AND PRELIMINARY}

Let $\mathbb{R}$ and $\mathbb{N}$ denote the reals and nonnegative integers, respectively. For some $r_{1} \in \mathbb{R}, n_{1}, n_{2} \in \mathbb{N}$ and $n_{2}>n_{1}, \mathbb{R}_{>r_{1}}$, $\mathbb{R}_{\geq r_{1}}, \mathbb{N}_{\geq n_{1}}, \mathbb{N}_{\leq n_{2}}$ and $\mathbb{N}_{\left[n_{1}, n_{2}\right]}$ denote the sets $\{r \in \mathbb{R} \mid r>$ $\left.r_{1}\right\},\left\{r \in \mathbb{R} \mid r \geq r_{1}\right\},\left\{n \in \mathbb{N} \mid n \geq n_{1}\right\},\left\{n \in \mathbb{N} \mid n \leq n_{2}\right\}$ and $\left\{n \in \mathbb{N} \mid n_{1} \leq n \leq n_{2}\right\}$, respectively. For a matrix $M$, its maximum and minimum eigenvalues are denoted by $\bar{\lambda}(M)$ and $\underline{\lambda}(M)$, respectively. For a symmetric matrix $P$, $P>0$ means that $P$ is positive definite. For a vector $x$, $\|x\| \triangleq \sqrt{x^{\mathrm{T}} x}$ and $\|x\|_{P} \triangleq \sqrt{x^{\mathrm{T} P x}}$ with $P>0$ represent the Euclidean norm and $P$-norm, respectively. Given two sets $\mathbb{A}$ and $\mathbb{B}$, their Minkowski set addition and Pontryagin set difference are denoted by $\mathbb{A} \oplus \mathbb{B} \triangleq\{a+b \mid a \in \mathbb{A}, b \in \mathbb{B}\}$ and $\mathbb{A} \ominus \mathbb{B} \triangleq\{a \mid\{a\} \oplus \mathbb{B} \subset \mathbb{A}\}$, respectively. $\mathbb{A} \backslash \mathbb{B}$ denotes the complementary set of $\mathbb{B}$ in $\mathbb{A}$. sup. $\in \mathbb{A}\{\cdot\}$ represents the upper bound of the elements in $\mathbb{A}$. The notations $x(k+i \mid k)$ and $u(k+i \mid k), k, i \in \mathbb{N}$, indicate the state and input prediction $i$ steps ahead from the current time $k$, respectively.

Before proceeding, we introduce the discrete GronwallBellman-Ou-Iang-type inequality.

Lemma 1. (See [8]) Let $\mathbb{S}=\left\{\mathbb{N}_{\geq m_{0}} \times \mathbb{N}_{\geq n_{0}}\right\}, \mu: \mathbb{S} \rightarrow \mathbb{R}_{>0}$, $b: \mathbb{S} \rightarrow \mathbb{R}_{>0}$, and let $\phi: \mathbb{R}_{>0} \rightarrow \mathbb{R}_{>0}$ be a continuous nondecreasing function. For $c \in \mathbb{R}_{\geq 0}, \alpha \in \mathbb{R}_{>0}$ and $(m, n) \in$ $\mathbb{S}$, if

$$
\mu^{\alpha}(m, n) \leq c+\sum_{s=m_{0}}^{m-1} \sum_{t=n_{0}}^{n-1} b(s, t) \phi(\mu(s, t))
$$

then

$$
\mu(m, n) \leq\left\{\Psi_{\alpha}^{-1}\left[\Psi_{\alpha}(c)+B(m, n)\right]\right\}^{\frac{1}{\alpha}}
$$

for all $m \leq m_{1}$ and $n \leq n_{1}$, where

$$
\Psi_{\alpha}(\gamma)=\int_{1}^{\gamma} \frac{d s}{\phi\left(s^{1 / \alpha}\right)}, \quad B(m, n)=\sum_{s=m_{0}}^{m-1} \sum_{t=n_{0}}^{n-1} b(s, t)
$$

$\Psi_{\alpha}^{-1}$ is the inverse of $\Psi_{\alpha}$, and $\left(m_{1}, n_{1}\right) \in \mathbb{S}$ is chosen such that $\Psi_{\alpha}(c)+B(m, n)$ is in the domain of $\Psi_{\alpha}^{-1}$ for all $m \leq m_{1}$ and $n \leq n_{1}$.

\section{PROBLEM SETUP}

\section{A. System definition}

Consider a general nonlinear system described by the following difference equation:

$$
x(k+1)=f(x(k), u(k))+w(k), \quad x(0)=x_{0},
$$

where $x(k) \in \mathbb{R}^{n}, u(k) \in \mathbb{R}^{m}$ and $w(k) \in \mathbb{R}^{n}$ are the system state, control input and external disturbance, respectively. It is assumed that the system is subject to the constraints

$$
x(k) \in \mathbb{X}, \quad u(k) \in \mathbb{U}, \quad w(k) \in \mathbb{W},
$$

where $\mathbb{X} \subset \mathbb{R}^{n}, \mathbb{U} \subset \mathbb{R}^{m}$ and $\mathbb{W}=\left\{w \in \mathbb{R}^{n}:\|w\| \leq \eta\right\}$ with $\eta \in \mathbb{R}_{>0}$ are compact sets containing the origin as an interior point. Furthermore, system (4) is assumed to satisfy the following conditions:

Assumption 1. The function $f(x(k), u(k)): \mathbb{R}^{n} \times \mathbb{R}^{m} \rightarrow \mathbb{R}^{n}$, with $f(\mathbf{0}, \mathbf{0})=\mathbf{0}$, is locally Lipschitz continuous in $x$. Let $g(x(k), u(k))=f(x(k), u(k))-x(k)$, and assume that $\ell$ is the Lipschitz constant of $g(x(k), u(k))$ with respect to $x$.

For expository reasons, we first define the nominal system by neglecting the disturbance:

$$
\hat{x}(k+1)=f(\hat{x}(k), \hat{u}(k)),
$$

where $\hat{x}(k) \in \mathbb{X}$ and $\hat{u}(k) \in \mathbb{U}$. Let $N \in \mathbb{N}_{\geq 1}$ be the prediction horizon. The cost function to be minimized online is given by

$$
\begin{aligned}
J(\hat{x}(k), \hat{u}(k), N)=\sum_{i=0}^{N-1} L(\hat{x}(k+ & i \mid k), \hat{u}(k+i \mid k)) \\
& +V_{N}(\hat{x}(k+N \mid k))
\end{aligned}
$$

with the stage cost $L(\hat{x}(k+i \mid k), \hat{u}(k+i \mid k))=\|\hat{x}(k+i \mid k)\|_{Q}^{2}+$ $\|\hat{u}(k+i \mid k)\|_{P}^{2}$ and the terminal cost $V_{N}(\hat{x}(k+N \mid k)), \hat{u}(k+$ $N \mid k))=\|\hat{x}(k+N \mid k)\|_{R}^{2}$. Here, $Q>0, P>0$ and $R>0$ are weighting matrices. Cost function (7) is a general form in MPC, see [9], [11] for detailed explanation of its physical meaning. To guarantee feasibility, the determination of the weighting matrices $P, Q$ and $R$ should meet the following assumption:

Assumption 2. There exist a set $\mathbb{X}_{r} \in \mathbb{X}$ with the form of $\mathbb{X}_{r}=\left\{\hat{x}: V_{N}(\hat{x}) \leq r^{2}\right\}$, a matrix $\Phi>0$ and a local stabilizing controller $\kappa(\hat{x}) \in \mathbb{U}$, such that, for all $\hat{x}(k) \in \mathbb{X}_{r}$, by implementing the controller $\kappa(\hat{x})$ it holds that

$$
\begin{gathered}
L(\hat{x}(k), \hat{u}(k)) \geq\|\hat{x}(k)\|_{\Phi}^{2}, \\
V_{N}(\hat{x}(k+1))-V_{N}(\hat{x}(k)) \leq-L(\hat{x}(k), \kappa(\hat{x}(k))) .
\end{gathered}
$$

It implies that $\mathbb{X}_{r}$ is a terminal region for system (6).

Remark 1. Assumptions 1 and 2 are fairly standard conditions to guarantee stability and feasibility of system (6) under MPC. How to choose a relatively weak conservative Lipschitz constant has been discussed in [10]. Moreover, several approaches to obtain the terminal region $\mathbb{X}_{r}$ and terminal controller $\kappa(\hat{x})$ have been proposed, such as by Jacobian linearization in [11], [12] and by feedback linearization in [13].

\section{B. Problem formulation}

The standard MPC setups in [1] and [11] lead to the closedloop system (6) meeting the performance guarantees of the form

$$
L(\hat{x}(k), \hat{u}(k)) \leq V(\hat{x}(k))-V(\hat{x}(k+1)),
$$

where $V(\hat{x}(k))$ is the optimal cost at $\hat{x}(k)$. However, the standard MPC requires solving an OCP at each update instant, 
which may require quite heavy computation. Moreover, the predictive horizon is usually a fixed constant. As a result, the controller has to solve the OCP with the same computational complexity at each step even when the system state gets close to the terminal region or the origin. Motivated by this, our control objective is to robustly stabilize system (4) to a set containing the origin while satisfying constraints (5), and at the same time, reducing the computation burden. In order to achieve this goal, a dual-mode MPC (see [14]) is implemented.

Before the system state enters the terminal region, a selftriggered MPC is adopted. Let $k_{j} \in \mathbb{N}$ be the update time, $N_{k_{j}}$ the prediction horizon at $k_{j}$, and $\hat{\boldsymbol{u}}^{*}\left(k_{j}\right)=$ $\left\{\hat{u}^{*}\left(k_{j} \mid k_{j}\right), \hat{u}^{*}\left(k_{j}+1 \mid k_{j}\right), \ldots, \hat{u}^{*}\left(k_{j}+N_{k_{j}}-1 \mid k_{j}\right)\right\}$ the optimal control sequence by solving the OCP at $k_{j}$. In system (4), the control input is given by

$$
u(k)=\hat{u}^{*}\left(k \mid k_{j}\right), \quad k \in \mathbb{N}_{\left[k_{j}, k_{j+1}-1\right]} .
$$

To reduce the frequency of solving the OCP, the update instant should be determined in a self-triggered fashion, i.e.,

$$
k_{j+1}=k_{j}+m\left(x\left(k_{j}\right)\right), \quad k_{0}=0,
$$

where $m(\cdot): \mathbb{R}^{n} \rightarrow \mathbb{N}_{\geq 1}$ denotes the inter-execution time. Meanwhile, the prediction horizon will adaptively decrease as the system state approaches the terminal region. This may reduce the computational complexity of the OCP. Specifically, the prediction horizon should be updated by

$$
N_{k_{j+1}}=N_{k_{j}}-n\left(x\left(k_{j}\right)\right), \quad N_{0}=N_{p},
$$

where $n(\cdot): \mathbb{R}^{n} \rightarrow \mathbb{N}$ represents the decreasing size of the prediction horizon, and $N_{p} \in \mathbb{N}_{\geq 1}$ is a given constant that guarantees feasibility of the OCP at the initial time. For ease of notation, we use $m_{k_{j}}$ and $n_{k_{j}}$ to denote $m\left(x\left(k_{j}\right)\right)$ and $n\left(x\left(k_{j}\right)\right)$, respectively. In addition, a sub-optimal performance

$$
\sum_{k=k_{j}}^{k_{j+1}} L\left(\hat{x}\left(k \mid k_{j}\right), \hat{u}\left(k \mid k_{j}\right)\right) \leq \beta\left(V\left(x\left(k_{j}\right)\right)-V\left(x\left(k_{j+1}\right)\right)\right)
$$

with $\beta>1$ will be still guaranteed.

Once the system state in (4) enters the terminal region, the controller will switch from solving the OCP to using $\kappa(x)$.

\section{ROBUST SELF-TRIGGERED MPC SCHEME WITH AN ADAPTIVE PREDICTION HORIZON}

In this section, a self-triggered MPC scheme with an adaptive prediction horizon is derived to robustly stabilize the system. The prediction horizon adaptively decreases as the system state approaches the terminal region, which will reduce the dimension of the OCP thereby reducing the computational complexity.

\section{A. Optimization control problem}

We first derive a difference bound between the nominal system and the actual one by an $m$-step, $m \in \mathbb{N}_{\geq 1}$, openloop control sequence with the same initial condition. This bound is provided by the following lemma.

Lemma 2. Denote an m-step control sequence as $\boldsymbol{u}_{m}=$ $\{u(k), u(k+1), \ldots, u(k+m-1)\}$. If the nominal system (6) and the perturbed one (4) are controlled by this sequence in an open-loop fashion enumerating from the same initial state, i.e., $x(k)=\hat{x}(k)$, then the state error defined by $x_{e}(k+m)=x(k+m)-\hat{x}(k+m)$ is norm-bounded:

$$
\left\|x_{e}(k+m)\right\|_{R} \leq m \eta \bar{\lambda}(\sqrt{R}) \exp \{\ell(m-1)\} .
$$

The proof of Lemma 2 is provided in Appendix A.

Based on the result in Lemma 2, we define a set sequence $\left\{\mathbb{X}_{e}(m), m=1,2, \ldots\right\}$, such that $x_{e}(k+m) \in \mathbb{X}_{e}(m)$ where

$$
\mathbb{X}_{e}(m)=\left\{\tilde{x}_{e}:\left\|\tilde{x}_{e}\right\|_{R} \leq m \eta \bar{\lambda}(\sqrt{R}) \exp \{\ell(m-1)\}\right\} .
$$

The OCP to be solved online is then formulated as follows.

OCP 1. At the update instant $k_{j}$, find the optimal control sequence $\hat{\boldsymbol{u}}^{*}\left(k_{j}\right)=\left\{\hat{u}^{*}\left(k_{j} \mid k_{j}\right), \hat{u}^{*}\left(k_{j}+1 \mid k_{j}\right), \ldots, \hat{u}^{*}\left(k_{j}+\right.\right.$ $\left.\left.N_{k_{j}}-1 \mid k_{j}\right)\right\}$ and the optimal state sequence $\hat{\boldsymbol{x}}^{*}\left(k_{j}\right)=$ $\left\{\hat{x}^{*}\left(k_{j}+1 \mid k_{j}\right), \hat{x}^{*}\left(k_{j}+2 \mid k_{j}\right), \ldots, \hat{x}^{*}\left(k_{j}+N_{k_{j}} \mid k_{j}\right)\right\}$ by solving the following minimization problem:

$$
\begin{aligned}
\hat{\boldsymbol{u}}^{*} & \left(k_{j}\right)=\arg \min _{\hat{\boldsymbol{u}}\left(k_{j}\right)} J\left(\hat{x}\left(k_{j}\right), \hat{u}\left(k_{j}\right), N_{k_{j}}\right) \\
& \hat{x}\left(k_{j} \mid k_{j}\right)=x\left(k_{j}\right), \\
& \hat{x}\left(k_{j}+i+1 \mid k_{j}\right)=f\left(\hat{x}\left(k_{j}+i \mid k_{j}\right), \hat{u}\left(k_{j}+i \mid k_{j}\right)\right), \\
\text { s.t. } & \hat{u}\left(k_{j}+i \mid k_{j}\right) \in \mathbb{U}, \\
& \hat{x}\left(k_{j}+i \mid k_{j}\right) \in \mathbb{X} \ominus \mathbb{X}_{e}(i), \\
& \hat{x}\left(k_{j}+N_{k_{j}} \mid k_{j}\right) \in \mathbb{X}_{\varepsilon},
\end{aligned}
$$

where $i \in \mathbb{N}_{\left[0, N_{k_{j}}-1\right]}$ and $\mathbb{X}_{\varepsilon}=\left\{\hat{x}:\|\hat{x}\|_{R}^{2} \leq \varepsilon^{2}\right\}$.

For OCP 1, we make the following mild assumption.

Assumption 3. OCP 1 is feasible at the initial time $k_{0}$ and $\mathbb{X}_{r} \subset \mathbb{X} \ominus \mathbb{X}_{e}\left(N_{p}\right)$.

The establishment condition of $\mathbb{X}_{r} \subset \mathbb{X} \ominus \mathbb{X}_{e}\left(N_{p}\right)$ will be discussed in Remark 3.

Remark 2. OCP 1 is a nonlinear programming problem, which can be solved by several algorithms, for example interior point approach [15], branch-and-bound optimization [16] and trust region reflective algorithm [17].

\section{B. Self-triggering mechanism and prediction horizon updating strategy}

In this subsection, we propose a self-triggering strategy and a prediction horizon update strategy. The triggering time is recursively calculated in terms of (12), and the open-loop phase $m_{k_{j}}$ is determined by the following condition:

$$
m_{k_{j}}=\min \left\{\hat{m}_{k_{j}}, \check{m}_{k_{j}}, N_{k_{j}}\right\}
$$

with

$$
\begin{array}{r}
\hat{m}_{k_{j}}=\sup \left\{m_{k_{j}}: m_{k_{j}} \eta \bar{\lambda}(\sqrt{R}) \exp \left\{\ell\left(N_{k_{j}}-1\right)\right\} \leq r-\varepsilon\right\}, \\
\check{m}_{k_{j}}=\sup \left\{m_{k_{j}}: m_{k_{j}} \eta \bar{\lambda}(\sqrt{R}) \exp \left\{\ell\left(N_{k_{j}}-1\right)\right\}(r+\varepsilon)\right. \\
+\sum_{i=m_{k_{j}}}^{N_{k_{j}}-1}\left[m_{k_{j}}^{2} \eta^{2} \bar{\lambda}^{2}(\sqrt{Q}) \exp \{2 \ell(i-1)\}\right. \\
\left.\quad+2 m_{k_{j}} \eta \bar{\lambda}(\sqrt{Q}) \exp \{\ell(i-1)\}\left\|\hat{x}^{*}\left(k_{j}+i \mid k_{j}\right)\right\|_{Q}\right] \\
\leq \sigma \sum_{i=0}^{m_{k_{j}}-1}\left(\left\|\hat{x}^{*}\left(k_{j}+i \mid k_{j}\right)\right\|_{Q}^{2}+\| \hat{u}^{*}\left(\left(k_{j}+i \mid k_{j}\right) \|_{P}^{2}\right)\right\},
\end{array}
$$


where $\sigma \in(0,1)$ is called performance factor. The prediction horizon is updated in terms of (13) and is decreased by

$$
n_{k_{j}}=\min \left\{m_{k_{j}}-1, N_{k_{j}}-\hat{N}_{k_{j}}\right\}
$$

where

$$
\hat{N}_{k_{j}}=\inf \left\{i: \hat{x}^{*}\left(k_{j}+i \mid k_{j}\right) \in \mathbb{X}_{\varepsilon}, i \in \mathbb{N}_{\left[0, N_{k_{j}-1}\right]}\right\} .
$$

The control sequence for the actual system over the interval $\left[k_{j}, k_{j+1}-1\right]$ is given by (11). The self-triggered MPC with adaptive prediction horizon scheme is then summarized in Algorithm 1.

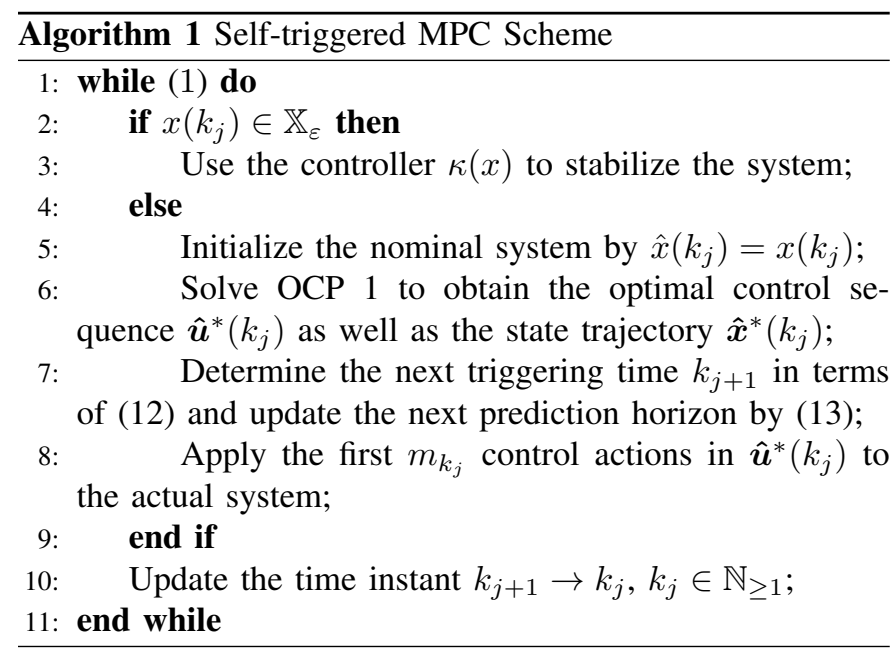

The development of self-triggered mechanism and horizon update strategy has physical meanings, and they are codesigned rather than a simple combination. On the one hand, increasing the open-loop control phase $m_{k_{j}}$ may enlarge the discrepancy between the predicted and the actual state trajectories. Condition (20) provided an upper bound of the discrepancy to guarantee recursive feasibility of the OCP. Since the open-loop control in the presence of external disturbances may deteriorate the convergence performance, (21) is derived to guarantee the sub-optimal performance (14). On the other hand, shrinking the prediction horizon may have potential advantages in reducing the computational complexity. However, a too short prediction horizon would not ensure stability of the closed-loop system. Therefore, condition (23) provides the minimum prediction horizon that can stabilize the system. Moreover, the decreased size of the prediction horizon should also be related to the open-loop phase, and the relationships are $1 \leq n_{k_{j}} \leq m_{k_{j}}-1$ and $1 \leq m_{k_{j}} \leq N_{k_{j}}$. Condition (22) implies that $k_{j+1}+N_{k_{j+1}} \geq k_{j}+N_{k_{j}}+1$. Furthermore, a balance between the triggering frequency and the optimal performance loss can be achieved by tuning the performance factor $\sigma$. Larger $\sigma$ can reduce the frequency of solving the OCP 1 and slow down the horizon update rate. Whereas smaller $\sigma$ can ensure a better closed-loop performance with a higher update frequency but slower changing rate of the horizon. Fig. 1 shows the connections between $m_{k_{j}}, N_{k_{j}}, \hat{N}_{k_{j}}$ and $N_{k_{j}}+1$. A more detailed analysis on (20) and (22) will be discussed in the next subsection.

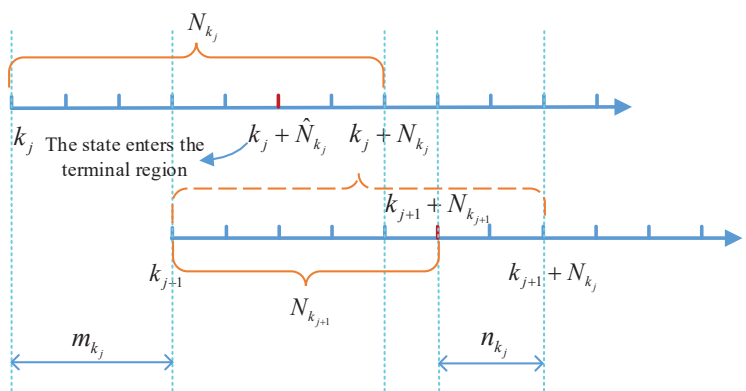

Fig. 1. Relationships between $m_{k_{j}}, N_{k_{j}}, \hat{N}_{k_{j}}$ and $N_{k_{j+1}}$.

\section{Analysis}

In this section, we analyze recursive feasibility of OCP 1 and stability of the closed-loop system under Algorithm 1. The following theorem shows recursive feasibility of OCP 1 .

Theorem 1. Suppose that the triggering time and prediction horizon are determined according to (19) and (22), respectively. OCP 1 is recursively feasible if $\left(1-\frac{\lambda(\Phi)}{\bar{\lambda}(R)}\right) r^{2} \leq \varepsilon^{2}$ and $\eta \leq \frac{r-\varepsilon}{\bar{\lambda}(\sqrt{R}) \exp \left\{\ell\left(N_{p}-1\right)\right\}}$.

Recursive feasibility implies that the solution space of OCP 1 is nonempty at each update time. For this, we first assume that an optimal control sequence is obtained at $k_{j}$ which is denoted by $\hat{\boldsymbol{u}}^{*}\left(k_{j}\right)=\left\{\hat{u}^{*}\left(k_{j} \mid k_{j}\right), \hat{u}^{*}\left(k_{j}+\right.\right.$ $\left.\left.1 \mid k_{j}\right), \ldots, \hat{u}^{*}\left(k_{j}+N_{k_{j}}-1 \mid k_{j}\right)\right\}$. The next update time is then determined by $k_{j+1}=k_{j}+m_{k_{j}}$ and the corresponding prediction horizon is updated by $N_{k_{j+1}}=N_{k_{j}}-n_{k_{j}}$. We construct a control sequence at $k_{j+1}$ as follows.

$\hat{u}\left(k_{j+1}+i \mid k_{j+1}\right)= \begin{cases}\hat{u}^{*}\left(k_{j+1}+i \mid k_{j}\right), & i \in \mathbb{N}_{\left[0, N_{k_{j}}-m_{k_{j}}-1\right]}, \\ \kappa\left(\hat{x}\left(k_{j+1}+i \mid k_{j}\right)\right), & i \in \mathbb{N}_{\left[N_{k_{j}}-m_{k_{j}}, N_{k_{j+1}}\right)} .\end{cases}$

We will show that (24) is a feasible solution to OCP 1 while satisfying all the constraints. Recursive feasibility of OCP 1 is then obtained by induction. The detailed proof of Theorem 1 is reported in Appendix B.

Remark 3. By substituting the upper bound of $\eta$ given by Theorem 1 into (16), we have $\mathbb{X}_{e}\left(N_{p}\right) \subseteq\left\{\tilde{x}_{e}:\left\|\tilde{x}_{e}\right\|_{R} \leq\right.$ $\left.N_{p}(r-\varepsilon)\right\}$. Therefore, $\mathbb{X} \supset\left\{\tilde{x}:\|\tilde{x}\|_{R} \leq N_{p}(r-\varepsilon)+r\right\}$ is a sufficient condition that guarantees $\mathbb{X}_{r} \subset \mathbb{X} \ominus \mathbb{X}_{e}\left(N_{p}\right)$ in Assumption 3.

Theorem 1 guarantees that OCP 1 is recursively feasible under some mild conditions. The following theorem establishes stability of system (4) under Algorithm 1.

Theorem 2. If the state of system (4) starts from the set $\mathbb{X} \backslash \mathbb{X}_{\varepsilon}$, then the system state under Algorithm 1 is guaranteed to enter the robust terminal region $\mathbb{X}_{\varepsilon}$ in finite time and to meet the suboptimal performance guarantee in (14) with $\beta=\frac{1}{1-\sigma}$. In addition, the system is ISS after the state enters $\mathbb{X}_{\varepsilon}$.

The proof of Theorem 2 involves two steps. In the first step, we assume that the system state starts from $\mathbb{X} \backslash \mathbb{X}_{\varepsilon}$ and will enter $\mathbb{X}_{\varepsilon}$ in finite time by examining optimal value of the cost function. In the second step, the system will be proven to be 

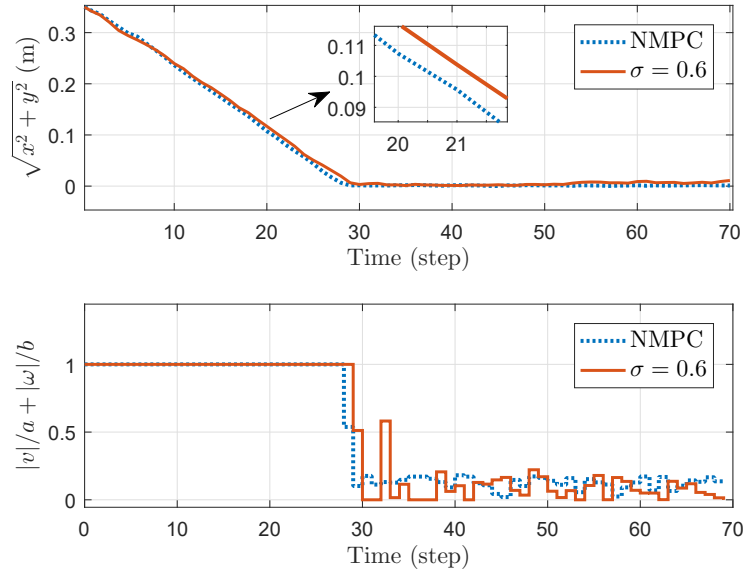

Fig. 2. Control error and input levels by the NMPC and the proposed approach with $\sigma=0.6$. The proposed approach reaches a satisfying performance.

ISS by the terminal controller. The detailed proof is reported in Appendix C.

\section{Simulation EXAmple}

In this section, we consider a position regulation problem of a nonholonomic vehicle. Its nominal system is described by the following dynamics:

$$
\left\{\begin{array}{l}
x(k+1)=x(k)+\delta v(k) \cos (\theta(k))-\delta \rho \omega(k) \sin (\theta(k)) \\
y(k+1)=y(k)+\delta v(k) \sin (\theta(k))+\delta \rho \omega(k) \cos (\theta(k)), \\
\theta(k+1)=\theta(k)+\delta \omega(k)
\end{array},\right.
$$

where $x, y$ and $\theta$ are the system states, $v$ and $\omega$ are the control inputs representing the linear and angular velocities, respectively, $\rho$ is the wheel base and $\delta$ is the sampling period. Then the system can be simply denoted as $\chi(k+1)=f(\chi(k), u(k))$ with $\chi=[x, y, \theta]^{\mathrm{T}}$ and $u=[v, \omega]^{\mathrm{T}}$. In the simulation, the wheel base and sampling period are set to be $\rho=0.0267 \mathrm{~m}$ and $\delta=0.1 \mathrm{~s}$, respectively. The input is constrained by $u \in\left\{u: \frac{v}{a}+\frac{\omega}{b} \leq 1\right\}$ with $a=0.13 \mathrm{~m} / \mathrm{s}$ and $b=4.8598 \mathrm{rad} / \mathrm{s}$. We assume that the disturbance on system (25) is bounded by $\eta=0.001$. We only consider the position regulation regardless of the orientation according to the design procedure in [18]. Then the weighting matrices in the cost function are set to be $Q=\operatorname{diag}\{0.06,0.06\}, P=\operatorname{diag}\{0.002,0.002\}$ and $R=\operatorname{diag}\{0.1,0.1\}$. The terminal controller is given as

$$
\left\{\begin{array}{l}
v(k)=\alpha_{1}[-x(k) \cos (\theta(k))-y(k) \sin (\theta(k))] \\
\omega(k)=\alpha_{2}[x(k) \sin (\theta(k))-y(k) \cos (\theta(k))] / \rho
\end{array}\right.
$$

where $\alpha_{1}=\alpha_{2}=0.6$. The terminal region parameters are then given by $r=0.0175$ and $\varepsilon=0.0128$.

To show the efficiency of the proposed scheme, we compare our results with nominal MPC (NMPC) in [13], i.e., using periodic sampling. We use distance $\sqrt{x^{2}+y^{2}}$ and control input constraints index $\frac{|v|}{a}+\frac{|\omega|}{b}$ to evaluate the control error and the control input levels. Fig. 2 shows the control error and control input levels by NMPC and the proposed selftriggered MPC with $\sigma=0.6$. It can be observed that the states by NMPC and self-triggered MPC with bounded uncertainties
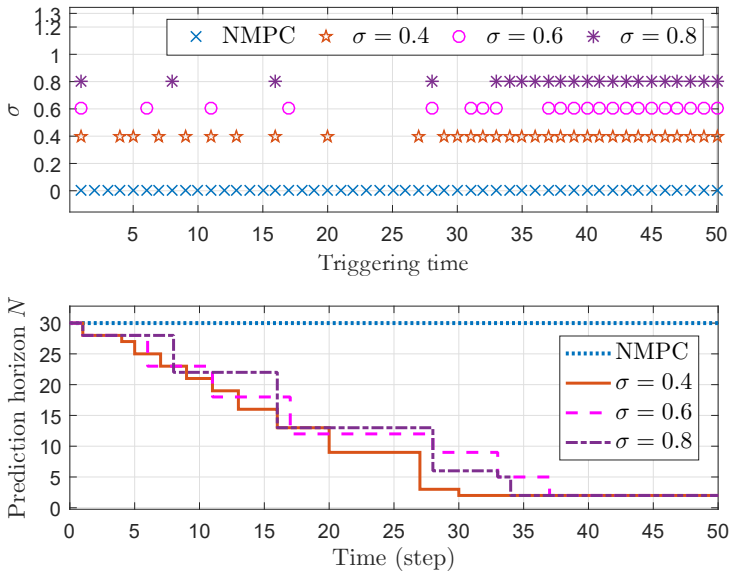

Fig. 3. Triggering times and prediction horizons by the NMPC and the proposed approach with $\sigma=0.4, \sigma=0.6$ and $\sigma=0.8$, respectively. The frequency of solving the OCP is reduced and the prediction horizon is decreasing as the system state is converging.
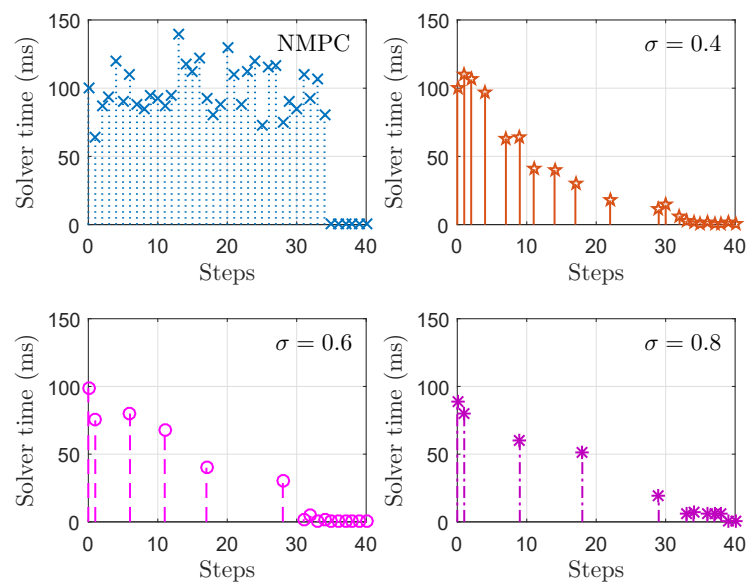

Fig. 4. The optimization times at each triggering instant by the NMPC and the proposed approach with $\sigma=0.4, \sigma=0.6$ and $\sigma=0.8$, respectively. The solver time is decreasing indicating that the computational complexity of the OCP is reduced by our approach.

converge to a neighbourhood of the origin, and the trajectories are almost the same. From the control input constraint index curve, we note that the control signals of NMPC are more smooth than the ones by self-triggered MPC. This is caused by the disturbances accumulation. Nevertheless, the input constraints are still ensured by the proposed scheme.

Next, we study how the parameter $\sigma$ in (21) affects the triggering time and prediction horizon. For this goal, we set the performance factor to be $\sigma=0.4, \sigma=0.6$ and $\sigma=0.8$ to observe the inter-execution time and the prediction horizon. Fig. 3 shows the triggering times (steps) by selftriggered MPC with different performance factors as well as by periodic NMPC. It can be seen that the inter-execution time increases as $\sigma$ is increasing, and the triggering time approaches periodic sampling as $\sigma$ gets close to zero. Meanwhile, the prediction horizon decreases as time goes by, which implies that the complexity of OCP 1 reduces since the number of 
TABLE I

THE TOTAL OPTIMIZATION TIMES BY NPMC AND SELF-TRIGGERED MPC WITH ADAPTIVE PREDICTION HORIZON

\begin{tabular}{|c|c|c|c|c|}
\hline Strategies & NMPC & \multicolumn{3}{|c|}{ Self-triggered MPC with adaptive prediction horizon } \\
\hline Parameters & $\delta=0.1 \mathrm{~s}$ & $\delta=0.1 \mathrm{~s}, \sigma=0.4$ & $\delta=0.1 \mathrm{~s}, \sigma=0.6$ & $\delta=0.1 \mathrm{~s}, \sigma=0.8$ \\
\hline Total optimization times $(\mathrm{ms})$ & 3466 & 714 & 409 & 335 \\
\hline
\end{tabular}

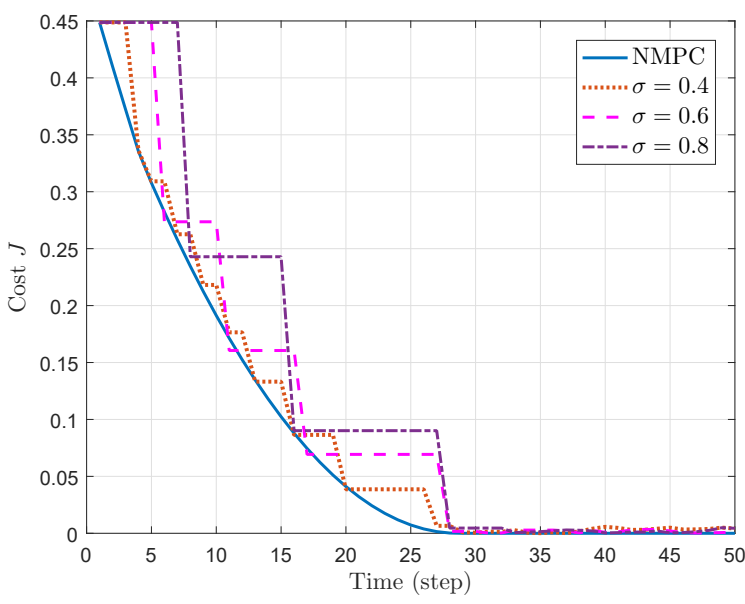

Fig. 5. Upper bounds of the cost by the NMPC and the proposed approach with $\sigma=0.4, \sigma=0.6$ and $\sigma=0.8$, respectively. These are lower bounded by the cost of the NMPC for the nominal system. The increase of $\sigma$ may enlarge the upper bound of the cost indicating that large $\sigma$ may degrade performance.

decision variables as well as the constraints decrease. This feature may facilitate practical application. It can also be seen that in all cases the triggering time becomes periodic at the end. This is because the system state has entered $\mathbb{X}_{\varepsilon}$ and the controller has switched to $\kappa(x)$ which is a periodic sampling control. Moreover, we use the optimization time to evaluate the computational complexity at each step. Fig. 4 shows the optimization times by NMPC and the proposed approach with different values of $\sigma$. In comparison to NMPC, the optimization time in each step is decreasing, indicating the reduction of computational complexity. The total optimization times are provided in Table I, which imply the computational benefit of the proposed approach. The cost upper bounds are depicted in Fig. 5, which verifies the suboptimal performance guarantee given by (14) with $\beta=\frac{1}{1-\sigma}$. This also indicates that the increase of $\sigma$ may deteriorate the performance.

\section{CONCLUSION}

We have proposed a robust self-triggered MPC with adaptive prediction horizon that robustly stabilizes the system while ensuring a suboptimal convergence performance. By designing a self-triggering mechanism and the prediction horizon update strategy, the average frequency of solving the OCP is reduced, and at the same time, the dimensionality of the OCP at each step is decreasing as the system state approaching the terminal region. This reduces the computational burden in the whole time-domain as well as the computational complexity at each update time. Rigorous analysis of recursive feasibility of the $\mathrm{OCP}$ and stability of the closed-loop system is conducted. Fi- nally, a simulation example is provided to show the theoretical results.

\section{APPENDIX A \\ PROOF OF LEMMA 2}

Proof. For the nominal system (6), we have the following recursive relations

$$
\hat{x}(k+i+1)-\hat{x}(k+i)=g(\hat{x}(k+i), u(k+i)),
$$

where $i \in \mathbb{N}_{[0, m-1]}$ and $g(\hat{x}(k+i), u(k+i))=f(\hat{x}(k+$ $i), u(k+i))-\hat{x}(k+i)$. Summing up (27) from $i=0$ to $i=m$ implies

$$
\hat{x}(k+m)=\hat{x}(k)+\sum_{i=0}^{m-1} g(\hat{x}(k+i), u(k+i)) .
$$

Similarly, for the perturbed system (4), we have

$$
x(k+m)=x(k)+\sum_{i=0}^{m-1}[g(x(k+i), u(k+i))+w(k+i)] .
$$

From (28) and (29), the state error can be written as

$$
\begin{gathered}
\left\|x_{e}(k+m)\right\|_{R}=\|x(k+m)-\hat{x}(k+m)\|_{R} \\
=\| x(k)+\sum_{i=0}^{m-1}[g(x(k+i), u(k+i))+w(k+i)] \\
\quad-\hat{x}(k)-\sum_{i=0}^{m-1} g(\hat{x}(k+i), u(k+i)) \|_{R} \\
\leq m \eta \bar{\lambda}(\sqrt{R})+\sum_{i=1}^{m-1} \ell\left\|x_{e}(k+i)\right\|_{R},
\end{gathered}
$$

where the condition $x(k)=\hat{x}(k)$ and the Lipschiz condition in Assumption 1 are used. If we apply Lemma 1 to (30) by letting $\mu(m, 1)=\left\|x_{e}(k+m)\right\|_{R}, \alpha=1, m_{0}=1, n=n_{0}+1$, $b(s, t)=\ell$ and $\phi(\mu)=\mu$, result (15) is straightforward.

\section{APPENDIX B}

\section{PROOF OF THEOREM 1}

Proof. At $k_{j}$, assume that an optimal control sequence is given by $\hat{\boldsymbol{u}}^{*}\left(k_{j}\right)=\left\{\hat{u}^{*}\left(k_{j} \mid k_{j}\right), \hat{u}^{*}\left(k_{j}+1 \mid k_{j}\right), \ldots, \hat{u}^{*}\left(k_{j}+\right.\right.$ $\left.\left.N_{k_{j}}-1 \mid k_{j}\right)\right\}$. The first $m_{k_{j}}$ control actions in $\hat{\boldsymbol{u}}^{*}\left(k_{j}\right)$, i.e., $\left\{\hat{u}^{*}\left(k_{j} \mid k_{j}\right), \hat{u}^{*}\left(k_{j}+1 \mid k_{j}\right), \ldots, \hat{u}^{*}\left(k_{j}+m_{k_{j}}-1 \mid k_{j}\right)\right\}$, are used to control the actual system. The open-loop control may lead to an error between the predicted and the actual state trajectories. It follows from Lemma 2 that this error is bounded by

$\left\|x\left(k_{j+1}\right)-\hat{x}^{*}\left(k_{j+1} \mid k_{j}\right)\right\|_{R} \leq m_{k_{j}} \eta \bar{\lambda}(\sqrt{R}) \cdot \exp \left\{\ell\left(m_{k_{j}}-1\right)\right\}$.

At time $k_{j+1}$, we construct a feasible solution as (24). Applying the control sequence $\hat{u}\left(k_{j+1}+i \mid k_{j+1}\right)=\hat{u}^{*}\left(k_{j+1}+i \mid k_{j}\right)$ 
over the interval $\left[k_{j+1}, k_{j}+N_{k_{j}}-1\right]$, the difference between the actual state and its prediction satisfies

$$
\begin{aligned}
& \left\|\hat{x}\left(k_{j+1}+i \mid k_{j+1}\right)-\hat{x}^{*}\left(k_{j+1}+i \mid k_{j}\right)\right\|_{R} \\
& \leq \| \sum_{s=0}^{i-1} g\left(\hat{x}\left(k_{j+1}+s \mid k_{j+1}\right), \hat{u}^{*}\left(k_{j+1}+s \mid k_{j}\right)\right) \\
& \quad-\sum_{s=0}^{i-1} g\left(\hat{x}^{*}\left(k_{j+1}+s \mid k_{j}\right), \hat{u}^{*}\left(k_{j+1}+s \mid k_{j}\right)\right) \|_{R} \\
& \quad+\left\|\hat{x}\left(k_{j+1} \mid k_{j+1}\right)-\hat{x}^{*}\left(k_{j+1} \mid k_{j}\right)\right\|_{R} \\
& \leq \sum_{s=0}^{i-1} \ell\left\|\hat{x}\left(k_{j+1}+s \mid k_{j+1}\right)-\hat{x}^{*}\left(k_{j+1}+s \mid k_{j}\right)\right\|_{R} \\
& \quad+\left\|\hat{x}\left(k_{j+1} \mid k_{j+1}\right)-\hat{x}^{*}\left(k_{j+1} \mid k_{j}\right)\right\|_{R},
\end{aligned}
$$

where the Lipschitz condition in Assumption 1 is used. Substituting $\hat{x}\left(k_{j+1} \mid k_{j+1}\right)=x\left(k_{j+1}\right)$ and (31) into (32) yields

$$
\begin{array}{r}
\left\|\hat{x}\left(k_{j+1}+i \mid k_{j+1}\right)-\hat{x}^{*}\left(k_{j+1}+i \mid k_{j}\right)\right\|_{R} \\
\leq \sum_{s=0}^{i-1} \ell\left\|\hat{x}\left(k_{j+1}+s \mid k_{j+1}\right)-\hat{x}^{*}\left(k_{j+1}+s \mid k_{j}\right)\right\|_{R} \\
+m_{k_{j}} \eta \bar{\lambda}(\sqrt{R}) \exp \left\{\ell\left(m_{k_{j}}-1\right)\right\} .
\end{array}
$$

It then follows from Lemma 1 that

$$
\begin{aligned}
\left\|\hat{x}\left(k_{j+1}+i \mid k_{j+1}\right)-\hat{x}^{*}\left(k_{j+1}+i \mid k_{j}\right)\right\|_{R} \\
\leq m_{k_{j}} \eta \bar{\lambda}(\sqrt{R}) \exp \left\{\ell\left(i+m_{k_{j}}-1\right)\right\} .
\end{aligned}
$$

Since $\eta \leq \frac{r-\varepsilon}{\bar{\lambda}(\sqrt{R}) \exp \left\{\ell\left(N_{p}-1\right)\right\}}$, there always exist $m_{k_{j}} \in$ $\mathbb{N}_{\left[1, N_{p}\right]}$ and $i \in \mathbb{N}_{\left[0, N_{k_{j}}-m_{k_{j}}\right]}$ such that $m_{k_{j}} \eta \bar{\lambda}(\sqrt{R}) \exp \{\ell(i+$ $\left.\left.m_{k_{j}}-1\right)\right\} \leq r-\varepsilon$ holds. Therefore, substituting $i=N_{k_{j}}-m_{k_{j}}$ into (34) and using the triangle inequality and (20), we get

$$
\left\|\hat{x}\left(k_{j}+N_{k_{j}} \mid k_{j+1}\right)\right\|_{R} \leq\left\|\hat{x}^{*}\left(k_{j}+N_{k_{j}} \mid k_{j}\right)\right\|_{R}+r-\varepsilon .
$$

Due to $\left\|\hat{x}^{*}\left(k_{j}+N_{k_{j}} \mid k_{j}\right)\right\|_{R} \leq \varepsilon$, we obtain

$$
\left\|\hat{x}\left(k_{j}+N_{k_{j}} \mid k_{j+1}\right)\right\|_{R} \leq r,
$$

which implies $\hat{x}\left(k_{j}+N_{k_{j}} \mid k_{j+1}\right) \in \mathbb{X}_{r}$. From Assumption 2, there exists a controller $\kappa(\hat{x})$ that can stabilize the nominal system and guarantee performance (9). Thus, utilizing the controller $\kappa\left(\hat{x}\left(k_{j+1}+i \mid k_{j}\right)\right)$ for $i \in \mathbb{N}_{\left[N_{k_{j}}-m_{k_{j}}, N_{k_{j+1}}-1\right]}$ yields

$$
\begin{array}{r}
V_{N}\left(\hat{x}\left(k_{j}+N_{k_{j}}+1 \mid k_{j+1}\right)\right)-V_{N}\left(\hat{x}\left(k_{j}+N_{k_{j}} \mid k_{j+1}\right)\right) \\
\leq-\left\|\hat{x}\left(k_{j}+N_{k_{j}} \mid k_{j+1}\right)\right\|_{\Phi}^{2},
\end{array}
$$

which implies

$$
\begin{aligned}
\| \hat{x}\left(k_{j}+N_{k_{j}}\right. & \left.+1 \mid k_{j+1}\right) \|_{R}^{2} \\
& \leq\left(1-\frac{\lambda}{\bar{\lambda}(R)}\right)\left\|\hat{x}\left(k_{j}+N_{k_{j}} \mid k_{j+1}\right)\right\|_{R}^{2} .
\end{aligned}
$$

Here $\frac{\lambda(\Phi)}{\lambda(R)}<1$ is guaranteed by the controller $\kappa(\hat{x})$. At time $k_{j+1}+N_{k_{j+1}}$, it holds that

$$
\begin{aligned}
& \left\|\hat{x}\left(k_{j+1}+N_{k_{j+1}} \mid k_{j+1}\right)\right\|_{R}^{2} \\
& \leq\left(1-\frac{\lambda}{\bar{\lambda}(\Phi)}\right)^{\left(N_{k_{j+1}}+m_{k_{j}}-N_{k_{j}}\right)}\left\|\hat{x}\left(k_{j}+N_{k_{j}} \mid k_{j+1}\right)\right\|_{R}^{2} .
\end{aligned}
$$

Since $1-\frac{\lambda(\Phi)}{\bar{\lambda}(R)} \leq \frac{\varepsilon^{2}}{r^{2}}$ and $N_{k_{j+1}}+m_{k_{j}}-N_{k_{j}} \geq 1$, we get

$$
\left\|\hat{x}\left(k_{j}+N_{k_{j}}+1 \mid k_{j+1}\right)\right\|_{R}^{2} \leq \varepsilon^{2},
$$

i.e., $\hat{x}\left(k_{j}+N_{k_{j}}+1 \mid k_{j+1}\right) \in \mathbb{X}_{\varepsilon}$. Now we have proved that the constructed control sequence (24) is able to drive the state into $\mathbb{X}_{\varepsilon}$ over the prediction horizon $N_{k_{j+1}}$.

Next, we show that input constraint and state constraint in (18) are guaranteed. From (24), the input constraint satisfaction is obvious. Thus, we only prove the satisfaction of state constraint. From (34), for $i \in \mathbb{N}_{\left[0, N_{k_{j}}-m_{k_{j}}-1\right]}$, we have

$$
\begin{aligned}
\left\|\hat{x}\left(k_{j+1}+i \mid k_{j+1}\right)\right\|_{R} \leq\left\|\hat{x}^{*}\left(k_{j+1}+i \mid k_{j}\right)\right\|_{R} \\
+m_{k_{j}} \eta \bar{\lambda}(\sqrt{R}) \exp \left\{\ell\left(i+m_{k_{j}}-1\right)\right\} .
\end{aligned}
$$

Since $\hat{x}^{*}\left(k_{j+1}+i \mid k_{j}\right) \in \mathbb{X} \ominus \mathbb{X}_{e}\left(i+m_{k_{j}}\right)$, thus

$$
\begin{aligned}
& \hat{x}\left(k_{j+1}+i \mid k_{j+1}\right) \in \mathbb{X} \ominus \mathbb{X}_{e}\left(i+m_{k_{j}}\right) \\
& \oplus m_{k_{j}} \eta \bar{\lambda}(\sqrt{R}) \exp \left\{\ell\left(i+m_{k_{j}}-1\right)\right\} \\
& \in \mathbb{X} \ominus \mathbb{X}_{e}(i), i \in \mathbb{N}_{\left[0, N_{k_{j}}-m_{k_{j}}-1\right]} .
\end{aligned}
$$

For $i \in \mathbb{N}_{\left[N_{k_{j}}-m_{k_{j}}, N_{k_{j+1}}-1\right]}$, the controller is switched to $\kappa\left(\hat{x}\left(k_{j+1}+i \mid k_{j}\right)\right)$, thus $\mathbb{X}_{r}$ is invariant. Since $N_{k_{j+1}} \leq N_{p}$ and $\mathbb{X}_{r} \in \mathbb{X} \ominus \mathbb{X}_{e}\left(N_{p}\right), \hat{x}\left(k_{j+1}+i \mid k_{j+1}\right) \in \mathbb{X} \ominus \mathbb{X}_{e}(i)$ is always satisfied. This completes the proof.

\section{APPENDIX C \\ PROOF OF THEOREM 2}

Proof. First assume that the system state starts from a point $x_{0} \in \mathbb{X} \backslash \mathbb{X}_{\varepsilon}$. Define a Lyapunov function as

$$
V\left(k_{j}\right)=J\left(\hat{x}^{*}\left(k_{j}\right), \hat{u}^{*}\left(k_{j}\right), N_{k_{j}}\right) .
$$

The difference of the Lyapunov function between the two successive instants $k_{j+1}$ and $k_{j}$ satisfies

$$
\begin{aligned}
& V\left(k_{j+1}\right)-V\left(k_{j}\right) \\
& \leq J\left(\hat{x}\left(k_{j+1}\right), \hat{u}\left(k_{j+1}\right), N_{k_{j+1}}\right)-J\left(\hat{x}^{*}\left(k_{j}\right), \hat{u}^{*}\left(k_{j}\right), N_{k_{j}}\right) \\
& \triangleq \Delta V_{1}+\Delta V_{2}+\Delta V_{3},
\end{aligned}
$$

where $\Delta V_{1}=-\sum_{i=0}^{m_{k_{j}}-1}\left(\left\|\hat{x}^{*}\left(k_{j}+i \mid k_{j}\right)\right\|_{Q}^{2}+\| \hat{u}^{*}\left(\left(k_{j}+i \mid k_{j}\right) \|_{P}^{2}\right)\right.$, $\Delta V_{2}=\sum_{i=m_{k_{j}}}^{N_{k_{j}}-1}\left(\left\|\hat{x}\left(k_{j}+i \mid k_{j+1}\right)\right\|_{Q}^{2}-\left\|\hat{x}^{*}\left(k_{j}+i \mid k_{j}\right)\right\|_{Q}^{2}\right)$, $\Delta V_{3}=\sum_{i=N_{k_{j}}}^{N_{k_{j}+1}+m_{k_{j}}-1}\left(\left\|\hat{x}\left(k_{j}+i \mid k_{j+1}\right)\right\|_{Q}^{2}+\| \hat{u}\left(k_{j}+\right.\right.$ $\left.\left.i \mid k_{j+1}\right) \|_{P}^{2}\right)+\left\|\hat{x}\left(k_{j+1}+N_{k_{j+1}} \mid k_{j+1}\right)\right\|_{R}^{2}-\left\|\hat{x}^{*}\left(k_{j}+N_{k_{j}} \mid k_{j}\right)\right\|_{R}^{2}$.

For $\Delta V_{2}$, using the result in (34) by replacing $R$ with $Q$, we have

$$
\begin{aligned}
& \Delta V_{2}=\sum_{i=m_{k_{j}}}^{N_{k_{j}}-1}\left(\left\|\hat{x}\left(k_{j}+i \mid k_{j+1}\right)\right\|_{Q}-\left\|\hat{x}^{*}\left(k_{j}+i \mid k_{j}\right)\right\|_{Q}\right) \\
& \quad \times\left(\left\|\hat{x}\left(k_{j}+i \mid k_{j+1}\right)\right\|_{Q}+\left\|\hat{x}^{*}\left(k_{j}+i \mid k_{j}\right)\right\|_{Q}\right) \\
& \leq \sum_{i=m_{k_{j}}}^{N_{k_{j}}-1}\left[m_{k_{j}}^{2} \eta^{2} \bar{\lambda}^{2}(\sqrt{Q}) \exp \{2 \ell(i-1)\}\right. \\
& \left.+2 m_{k_{j}} \eta \bar{\lambda}(\sqrt{Q}) \exp \{\ell(i-1)\}\left\|\hat{x}^{*}\left(k_{j}+i \mid k_{j}\right)\right\|_{Q}\right] .
\end{aligned}
$$


Then consider $\Delta V_{3}$, which can be rewritten as

$$
\begin{gathered}
\Delta V_{3}=\sum_{i=N_{k_{j}}}^{N_{k_{j+1}+m_{k_{j}}-1}}\left(\left\|\hat{x}\left(k_{j}+i \mid k_{j+1}\right)\right\|_{Q}^{2}+\left\|\hat{u}\left(k_{j}+i \mid k_{j+1}\right)\right\|_{P}^{2}\right) \\
+\left\|\hat{x}\left(k_{j+1}+N_{k_{j+1}} \mid k_{j+1}\right)\right\|_{R}^{2}-\left\|\hat{x}\left(k_{j}+N_{k_{j}} \mid k_{j+1}\right)\right\|_{R}^{2} \\
+\left\|\hat{x}\left(k_{j}+N_{k_{j}} \mid k_{j+1}\right)\right\|_{R}^{2}-\left\|\hat{x}^{*}\left(k_{j}+N_{k_{j}} \mid k_{j}\right)\right\|_{R}^{2} . \quad(44)
\end{gathered}
$$

By virtue of (9), we have the following relationships:

$$
\begin{aligned}
& \left\|\hat{x}\left(k_{j+1}+N_{k_{j+1}} \mid k_{j+1}\right)\right\|_{R}^{2}-\left\|\hat{x}\left(k_{j+1}+N_{k_{j+1}}-1 \mid k_{j+1}\right)\right\|_{R}^{2} \\
& \leq-\left\|\hat{x}\left(k_{j+1}+N_{k_{j+1}}-1 \mid k_{j+1}\right)\right\|_{Q}^{2}-\left\|\hat{u}\left(k_{j+1}+N_{k_{j+1}}-1 \mid k_{j+1}\right)\right\|_{P}^{2}, \\
& \left\|\hat{x}\left(k_{j+1}+N_{k_{j+1}}-1 \mid k_{j+1}\right)\right\|_{R}^{2}-\left\|\hat{x}\left(k_{j+1}+N_{k_{j+1}}-2 \mid k_{j+1}\right)\right\|_{R}^{2} \\
& \leq-\left\|\hat{x}\left(k_{j+1}+N_{k_{j+1}}-2 \mid k_{j+1}\right)\right\|_{Q}^{2}-\left\|\hat{u}\left(k_{j+1}+N_{k_{j+1}}-2 \mid k_{j+1}\right)\right\|_{P}^{2}, \\
& \ldots \\
& \left\|\hat{x}\left(k_{j}+N_{k_{j}}+1 \mid k_{j+1}\right)\right\|_{R}^{2}-\left\|\hat{x}\left(k_{j}+N_{k_{j}} \mid k_{j+1}\right)\right\|_{R}^{2} \\
& \leq-\left\|\hat{x}\left(k_{j}+N_{k_{j}} \mid k_{j+1}\right)\right\|_{Q}^{2}-\left\|\hat{u}\left(k_{j}+N_{k_{j}} \mid k_{j+1}\right)\right\|_{P}^{2} .
\end{aligned}
$$

Summing up (45) yields

$$
\begin{aligned}
& \sum_{i=N_{k_{j}}}^{N_{k_{j+1}}+m_{k_{j}}-1}\left(\left\|\hat{x}\left(k_{j}+i \mid k_{j+1}\right)\right\|_{Q}^{2}+\left\|\hat{u}\left(k_{j}+i \mid k_{j+1}\right)\right\|_{P}^{2}\right) \\
& +\left\|\hat{x}\left(k_{j+1}+N_{k_{j+1}} \mid k_{j+1}\right)\right\|_{R}^{2}-\left\|\hat{x}\left(k_{j}+N_{k_{j}} \mid k_{j+1}\right)\right\|_{R}^{2} \leq 0 .
\end{aligned}
$$

Substituting (46) into (44), we have

$$
\begin{aligned}
\Delta V_{3} \leq & \left\|\hat{x}\left(k_{j}+N_{k_{j}} \mid k_{j+1}\right)\right\|_{R}^{2}-\left\|\hat{x}^{*}\left(k_{j}+N_{k_{j}} \mid k_{j}\right)\right\|_{R}^{2} \\
\leq & \left(\left\|\hat{x}\left(k_{j}+N_{k_{j}} \mid k_{j+1}\right)\right\|_{R}-\left\|\hat{x}^{*}\left(k_{j}+N_{k_{j}} \mid k_{j}\right)\right\|_{R}\right) \\
& \times\left(\left\|\hat{x}\left(k_{j}+N_{k_{j}} \mid k_{j+1}\right)\right\|_{R}+\left\|\hat{x}^{*}\left(k_{j}+N_{k_{j}} \mid k_{j}\right)\right\|_{R}\right) \\
\leq & m_{k_{j}} \eta \bar{\lambda}(\sqrt{R}) \exp \left\{\ell\left(N_{k_{j}}-1\right)\right\}(r+\varepsilon) .
\end{aligned}
$$

Combining (42) with (43) and (47) yields

$$
\begin{aligned}
& V\left(k_{j+1}\right)-V\left(k_{j}\right) \\
& \leq-\sum_{i=0}^{m_{k_{j}}-1}\left(\left\|\hat{x}^{*}\left(k_{j}+i \mid k_{j}\right)\right\|_{Q}^{2}+\| \hat{u}^{*}\left(\left(k_{j}+i \mid k_{j}\right) \|_{P}^{2}\right)\right. \\
& +\sum_{i=m_{k_{j}}}^{N_{k_{j}}-1}\left[m_{k_{j}}^{2} \eta^{2} \bar{\lambda}^{2}(\sqrt{Q}) \exp \{2 \ell(i-1)\}\right. \\
& \left.+2 m_{k_{j}} \eta \bar{\lambda}(\sqrt{Q}) \exp \{\ell(i-1)\}\left\|\hat{x}^{*}\left(k_{j}+i \mid k_{j}\right)\right\|_{Q}\right] \\
& \quad+m_{k_{j}} \eta \bar{\lambda}(\sqrt{R}) \exp \left\{\ell\left(N_{k_{j}}-1\right)\right\}(r+\varepsilon) .
\end{aligned}
$$

Specifically, since self-triggered condition (21) is satisfied at $k_{j+1}$, it follows that

$$
\begin{aligned}
& V\left(k_{j+1}\right)-V\left(k_{j}\right) \\
& \leq(\sigma-1) \sum_{i=0}^{m_{k_{j}}-1}\left(\left\|\hat{x}^{*}\left(k_{j}+i \mid k_{j}\right)\right\|_{Q}^{2}+\left\|\hat{u}^{*}\left(k_{j}+i \mid k_{j}\right)\right\|_{P}^{2}\right) \\
& \leq(\sigma-1)\left\|\hat{x}^{*}\left(k_{j} \mid k_{j}\right)\right\|_{\Phi}^{2} \leq(\sigma-1) \frac{\lambda(\Phi)}{\bar{\lambda}(R)} \varepsilon^{2} .
\end{aligned}
$$

This guarantees the suboptimal performance in (14) with $\beta=$ $\frac{1}{1-\sigma}$. Moreover, by induction we get $V\left(k_{j+1}\right) \leq V(0)-(j+$ 1) $(1-\sigma) \frac{\lambda\left(\frac{\lambda}{\bar{\lambda}}(R)\right.}{2} \varepsilon^{2}$. Therefore it can be concluded that the system state will enter $\mathbb{X}_{\varepsilon}$ in finite time by following the argument in [14, Theorem 2].

When $x \in \mathbb{X}_{\varepsilon}$, the controller will be switched to $\kappa(x)$. Taking $V_{N}(x)$ as a Lyapunov function, we have

$$
\begin{gathered}
V_{N}(x(k+1))-V_{N}(x(k)) \\
\leq\|f(x(k), \kappa(x(k)))+w(k)\|_{R}^{2}-\|x(k)\|_{R}^{2} \\
\leq\|f(x(k), \kappa(x(k)))\|_{R}^{2}-\|x(k)\|_{R}^{2} \\
\quad+2 w^{\mathrm{T}} R f\left(x(k), \kappa(x(k))+\|w(k)\|_{R}^{2}\right. \\
\leq-\|x(k)\|_{\Phi}^{2}+2 \eta \varepsilon \frac{\bar{\lambda}(\sqrt{R})}{\underline{\lambda}(\sqrt{R})}+\bar{\lambda}(R) \eta^{2}
\end{gathered}
$$

which constitutes an ISS-Lyapunov function.

\section{REFERENCES}

[1] D. Q. Mayne, J. B. Rawlings, C. V. Rao, and P. O. M. Scokaert, "Constrained model predictive control: Stability and optimality," Automatica, vol. 36, no. 6, pp. 789-814, 2000.

[2] Y. Iino, T. Hatanaka, and M. Fujita, "Event-predictive control for energy saving of wireless networked control system," in Proceedings of American Control Conference, 2009, pp. 2236-2242.

[3] H. Li and Y. Shi, "Event-triggered robust model predictive control of continuous-time nonlinear systems," Automatica, vol. 50, no. 5, pp. $1507-1513,2014$.

[4] T. Gommans, D. Antunes, T. Donkers, P. Tabuada, and M. Heemels, "Self-triggered linear quadratic control," Automatica, vol. 50, no. 4, pp. 1279-1287, 2014.

[5] F. D. Brunner, M. Heemels, and F. Allgöwer, "Robust self-triggered MPC for constrained linear systems: A tube-based approach," Automatica, vol. 72, pp. 73-83, 2016.

[6] K. Hashimoto, S. Adachi, and D. V. Dimarogonas, "Self-triggered model predictive control for nonlinear input-affine dynamical systems via adaptive control samples selection," IEEE Transactions on Automatic Control, vol. 62, no. 1, pp. 177-189, 2017.

[7] — , "Event-triggered intermittent sampling for nonlinear model predictive control," Automatica, vol. 81, pp. 148-155, 2017.

[8] W. Cheung and J. Ren, "Discrete non-linear inequalities and applications to boundary value problems," Journal of Mathematical Analysis and Applications, vol. 319, no. 2, pp. 708-724, 2006.

[9] J. B. Rawlings and D. Q. Mayne, Model predictive control: Theory and design. Nob Hill Pub., 2009.

[10] D. L. Marruedo, T. Alamo, and E. F. Camacho, "Input-to-state stable MPC for constrained discrete-time nonlinear systems with bounded additive uncertainties," in Proceedings of Conference on Decision and Control, vol. 4, 2002, pp. 4619-4624.

[11] H. Chen and F. Allgöwer, "A quasi-infinite horizon nonlinear model predictive control scheme with guaranteed stability," Automatica, vol. 34, no. 10, pp. 1205-1217, 1998.

[12] M. Althoff, O. Stursberg, and M. Buss, "Reachability analysis of nonlinear systems with uncertain parameters using conservative linearization," in Proceedings of Conference on Decision and Control, 2008, pp. 40424048.

[13] Z. Sun and Y. Xia, "Receding horizon tracking control of unicycle-type robots based on virtual structure," International Journal of Robust and Nonlinear Control, vol. 26, no. 17, pp. 3900-3918, 2016.

[14] H. Michalska and D. Q. Mayne, "Robust receding horizon control of constrained nonlinear systems," IEEE Transactions on Automatic Control, vol. 38, no. 11, pp. 1623-1633, 1993.

[15] A. Wächter and L. T. Biegler, "On the implementation of an interiorpoint filter line-search algorithm for large-scale nonlinear programming," Mathematical Programming, vol. 106, no. 1, pp. 25-57, 2006.

[16] P. M. Narendra and K. Fukunaga, "A branch and bound algorithm for feature subset selection," IEEE Transactions on Computers, vol. 26, no. 9, pp. 917-922, 1977.

[17] T. F. Coleman and Y. Li, "An interior trust region approach for nonlinear minimization subject to bounds," SIAM Journal on optimization, vol. 6 , no. 2, pp. 418-445, 1996.

[18] Z. Sun, L. Dai, K. Liu, Y. Xia, and K. H. Johansson, "Robust MPC for tracking constrained unicycle robots with additive disturbances," Automatica, vol. 90, pp. 172-184, 2018. 